\title{
On Straneo's Unified Theory of Gravitation and Electricity
}

By P. G. Gormley, Edinburgh Cniversity.

(Received 24th January, 1933. Read 3rd March, 1933.)

In a recent series of papers, 1 Straneo has introduced into Riemannian space-time a teleparallelism defined by an asymmetric connection, and in this manner attempted to develop a unified theory of gravitation and electricity. The essential idea underlying his work is, therefore, similar to that of the Einstein-Maver theory of 1929-31.

In this paper, it is shown that the field equations proposed by Straneo have only a limited number of solutions. Thus they cannot be the correct field equations for a general electromagnetic field. There are in fact three distinct solutions, one the Galilean world, one the Einstein cylindrical world, while the other does not appear to be any well known space-time.

$\S 1$. Summary of Straneo's Theory.

This section contains the essence of Straneo's theory. His notation, though not the mode of presentation is preserved.

In a Riemannian space-time of four dimensions with the metric ${ }^{2}$

let

$$
d s^{2}=g_{\mu \nu} d x^{\mu} d x^{\nu},
$$

$$
L_{\lambda \mu}^{\nu} \equiv \Omega_{. \lambda \mu}^{\nu}+\left\{\begin{array}{l}
\nu \\
\lambda \mu
\end{array}\right\},
$$

where $\left\{\begin{array}{l}\nu \\ \lambda \mu\end{array}\right\}$ are the Christoffel symbols and $\Omega_{. \lambda \mu}^{\nu}$ is the torsion tensor, define the asymmetric connection. The connection is defined to have the following properties:

(i) it is a teleparallelism,

(ii) its autoparallels are the geodesics of the Riemannian space,

(iii) the length of a vector is unchanged by parallel transport,

(iv) the angle between any two directions at a point is unchanged when these directions are transferred by this parallel transport to

1 ZS. f. Phys., 77 (1932), 829. Also Rendiconti dei Lincei, 15 (1932), 462 and 563, $16(1932 \cdot 3), 139$.

2 The summation convention is observed throughout this paper. 
any other point, so long as the curves along which the vectors have been transferred can be made to coincide with each other by continuous deformation.

The properties (i), (ii), (iii) are quite sufficient to determine this connection which is a generalisation of Clifford parallelism ${ }^{1}$ in the elliptic space of three dimensions.

It is easily shown that the properties (ii) and (iii) give respectively

and hence

$$
\begin{aligned}
& \Omega_{. \lambda \mu}^{\nu}+\Omega_{. \mu \lambda}^{\nu}=0, \\
& \Omega_{\nu \lambda \mu}+\Omega_{\lambda \nu \mu}=0,
\end{aligned}
$$

Then since the connection (1.1) defines a teleparallelism we have

$$
L_{. \lambda \mu \rho}^{\nu}=\underset{\delta \overline{x^{\mu}}}{\partial} L_{\lambda \rho}^{\nu}-\frac{\partial}{\partial x^{\rho}} L_{\lambda \mu}^{\nu}+L_{\lambda \rho}^{\epsilon} L_{\epsilon \mu}^{\nu}-L_{\lambda \mu}^{e} L_{\varepsilon \rho}^{\nu}=0
$$

whence we find

$$
R_{. \lambda \mu \rho}^{v}+\Omega_{. \lambda \rho, \mu}^{\nu}-\Omega_{. \lambda \mu, \rho}^{\nu}+\Omega_{. \lambda \rho}^{a} \Omega_{. \alpha \mu}^{\nu}-\Omega_{. \lambda \mu}^{a} \Omega_{. \alpha \rho}^{v}=0 .
$$

Then permuting the suffixes $\lambda \mu \rho$ cyclically, noting the cyclic property of the Riemann-Christoffel tensor, we find on substituting the identity so found in this last equation, the equation

$$
R_{. \lambda \mu \rho}^{v}+\Omega_{. \mu \rho, \lambda}^{v}+\Omega_{. \mu \rho}^{\alpha} \Omega_{. \alpha \lambda}^{\nu}=0 .
$$

Lowering the suffix $\nu$ we have

Since by (1.4)

$$
R_{\nu \lambda \mu \rho}+\Omega_{\nu \mu \rho, \lambda}+\Omega_{. \mu \rho}^{a} \Omega_{v a \lambda}=0 .
$$

$$
\Omega_{\nu \mu \rho}=\frac{1}{; 3}\left(\Omega_{\nu \mu \rho}+\Omega_{\mu \rho \nu}+\Omega_{\rho \nu \mu}\right) \text {, }
$$

we find from (1.7) by permuting cyclically the suffixes $\nu \mu \rho$, and adding the expressions thus found, the relation

$$
\Omega_{\nu \mu \rho, \lambda}+\frac{1}{i 3}\left(\Omega_{. \mu \rho}^{a} \Omega_{\nu a \lambda}+\Omega_{.{ }^{\prime} \nu}^{a} \Omega_{\mu a \lambda}+\Omega_{.{ }_{\nu \mu}}^{a} \Omega_{\rho \alpha \lambda}\right)=0 .
$$

Then equations (1.7) become simply

$$
R_{\nu \lambda \mu \rho}=\frac{1}{i ;}\left(\Omega_{\cdot{ }_{\rho \nu}}^{a} \Omega_{\mu a \lambda}+\Omega_{. \nu \mu}^{a} \Omega_{\rho a \lambda}-2 \Omega_{. \mu \rho}^{a} \Omega_{r a \lambda}\right) .
$$

By equations (1.2) (1.3) and (1.8), it is evident that the tensor $\Omega_{r_{\mu \rho, ~} \text { A }}$ is skew in all its suffixes. It is easily proved that

If the Riemann-Christoffel tensor can be expressed in the form (1.9) where the tensor $\Omega_{\nu \mu \lambda}$ is skew in all its suffixes, and also satisfies the equations (1.8), then the space admits the absolute parallelism defined by (1.1).

1 cf. Whittaker, Joumal London Math. Soc., 5 (1930), 68-80, especially pages 74-79. 


\section{$\S 2$. Consequences of the Field Equations.}

Straneo takes the field equations to be (1.6), which are equivalent to (1.9). The torsion tensor $\Omega_{. \lambda \mu}^{\nu}$ must satisfy the equations (1.8). Since we are dealing with a space of four dimensions, the skew tensor $\Omega_{\nu \lambda \mu}$ has only four independent components, while the skew tensor $\Omega_{\nu \lambda \mu, \rho}$ has only one independent component. Thus we have

$$
\Omega_{\nu \lambda \mu \rho}=A \epsilon_{\nu \lambda \mu \rho}
$$

where $A$ is a scalar, and $\epsilon_{\nu \lambda \mu \rho}$ the tensor which has the value zero when any two of $\nu \lambda \mu \rho$ are equal, and the value $\pm \sqrt{ }(-g)$ according as $\nu \lambda \mu \rho$ is an even or odd permutation of $0,1,2,3$.

If we define a vector $\psi^{\lambda}$ as follows

then we have

$$
6 \psi^{\lambda}=\epsilon^{\lambda \mu \nu \sigma} \Omega_{\mu \nu \sigma},
$$

$$
\Omega_{\mu \nu v}=\epsilon_{\lambda \mu \nu v} \psi^{\lambda} \text {. }
$$

Substituting from (2.1) and (2.3) in (1.8) we have

$$
A \epsilon_{\nu \mu \rho \lambda}+\frac{1}{3} g^{a \beta} \psi^{\sigma} \psi^{r}\left(\epsilon_{\sigma \beta \mu \rho} \epsilon_{\tau \nu \alpha \lambda}+\epsilon_{\sigma \beta \rho \nu} \epsilon_{\tau \mu \alpha \lambda}+\epsilon_{\sigma \beta \nu \mu} \epsilon_{\tau \rho \alpha \lambda}\right)=0 .
$$

Then multiplying by $\epsilon^{\nu \mu \rho \lambda}$ and summing for $\nu \mu \rho$ and $\lambda$ it is readily seen that

and hence

$$
A=0 \text {, }
$$

$$
\Omega_{\nu \mu \rho, \lambda}=\mathbf{0} \text {. }
$$

Thus, by (1.6) and (2.5) we have

$$
R_{. \lambda \mu \rho}^{\nu}+\Omega_{. \mu \rho}^{a} \Omega_{. a \lambda}^{\nu}=0,
$$

while equation (1.8) is satisfied identically because of the cyclic property of the Riemann-Christoffel tensor. Then from (2.6) it is evident that the space admits the two teleparallelisms defined by

$$
L_{\lambda \mu}^{\nu}=\left\{\begin{array}{l}
\nu \\
\lambda \mu
\end{array}\right\} \pm \Omega_{. \lambda \mu}^{\nu}
$$

From (2.5) and (2.6) it is easily shown that

whence

$$
R_{. \lambda \mu \rho, \sigma}^{\nu}=0 \text {, }
$$

$$
\partial R / \partial x^{\sigma}=0, \text { where } R=g^{\lambda \mu} R_{. \lambda \mu \nu}^{\nu} .
$$

The scalar curvature of the space is therefore everywhere constant. 
Differentiating the equation (2.2) covariantly, and using equation (2.5), we find that

and hence

$$
\psi_{, p}^{\lambda}=0 \text {, }
$$

Thus we have

$$
\psi_{\mu, \nu}=0 \text {. }
$$

$$
\psi_{\mu, \nu}-\psi_{\nu, \mu}=\frac{\partial \psi_{\mu}}{\partial x^{\nu}}-\frac{\partial \psi_{v}}{\partial x^{\mu}}=0
$$

and hence $\psi_{\mu} d x^{\mu}$ is a perfect differential $d \psi$, so we have

$$
\psi_{\mu}=\frac{\hat{z} \psi}{\partial x^{\mu}},
$$

where $\psi$ is a scalar function satisfying the equation

$$
\psi_{, \mu \nu}=\frac{\partial^{2} \psi}{\partial x^{\mu} \partial x^{\nu}}-\left\{\begin{array}{l}
\sigma \\
\mu \nu
\end{array}\right\} \frac{\partial \psi}{\partial x^{\sigma}}=0 .
$$

Now equation (2.6) can be written

$$
R_{. . \mu \rho}^{\nu \lambda}=\Omega^{a \nu \lambda} \Omega_{a \mu \rho}
$$

Then because of equation (2.3), this last equation becomes

$$
R_{., \mu \rho}^{\nu \lambda}=\delta_{\mu \rho}^{\nu \lambda} \psi_{\sigma} \psi^{\sigma}+\psi_{\mu} \psi^{\lambda} \delta_{\rho}^{\nu}-\psi_{\mu} \psi^{\nu} \delta_{\rho}^{\lambda}+\psi_{\rho} \psi^{\nu} \delta_{\mu}^{\lambda}-\psi_{\rho} \psi^{\lambda} \delta_{\mu}^{\nu},
$$

whence

$$
\begin{gathered}
R_{. \lambda \mu \rho}^{v}=\left(\delta_{\mu}^{v} g_{\lambda_{\rho}}-\delta_{\rho}^{v} g_{\lambda \mu}\right) \psi_{\sigma} \psi^{\sigma}+\psi_{\mu} \psi_{\lambda} \delta_{\rho}^{\nu}-\psi_{\mu} \psi^{v} g_{\lambda \rho}+\psi_{\rho} \psi^{v} g_{\lambda \mu} \\
-\psi_{\rho} \psi_{\lambda} \delta_{\mu}^{v} .
\end{gathered}
$$

\section{§3. Soldtion of the Field Equations-I.}

In the present section it is shown that the space-time for which Straneo's theory is valid are conformally representable on a flat space-time.

Consider the space with fundamental tensor

$$
\gamma_{\mu \lambda}=e^{-2 \psi} g_{\mu \lambda},
$$

and denote the Christoffel symbols for this space by $\left\{\begin{array}{l}\nu \\ \mu \lambda\end{array}\right\}$. Then ${ }^{1}$

$$
\left\{\begin{array}{l}
\nu \\
\mu \lambda
\end{array}\right\}=\left\{\begin{array}{l}
\nu \\
\mu \lambda
\end{array}\right\}-\left(\delta_{\mu}^{\nu} \psi_{\lambda}+\delta_{\lambda}^{\nu} \psi_{\mu}-g_{\lambda \mu} g^{\nu \sigma} \psi_{\sigma}\right) \text {. }
$$

On forming the Riemann-Christoffel tensor, remembering that equation (2.10) is satisfied, we find

$$
\begin{gathered}
R_{. \lambda \mu \rho}^{\nu}=R_{. \lambda \mu \rho}^{\nu}+\delta_{\mu}^{\nu}\left(\psi_{\lambda} \psi_{\rho}-g_{\lambda \rho} \psi_{\sigma} \psi^{\sigma}\right)-\delta_{\rho}^{\nu}\left(\psi_{\lambda} \psi_{\mu}-g_{\lambda \mu} \psi_{\sigma} \psi^{\sigma}\right) \\
+g_{\lambda \rho} \psi_{\mu} \psi^{\nu}-g_{\lambda \mu} \psi_{\rho} \psi^{\nu} .
\end{gathered}
$$

, 1 Cf. Eisenhart. Riemannian Geometry, 89. 
Using equation (2.14) we find that

$$
' R_{. \lambda \mu \rho}^{\nu}=0 \text {. }
$$

The $V_{4}$ is therefore conformal to a flat space.

By choosing a suitable system of coordinates we can, then, make and so

$$
\gamma_{\mu \nu} d x^{\mu} d x^{\nu} \equiv\left(d x^{0}\right)^{2}-\left(d x^{1}\right)^{2}-\left(d x^{2}\right)^{2}-\left(d x^{3}\right)^{2},
$$

$$
d s^{2}=g_{\mu \nu} d x^{\mu} d x^{\nu}=e^{2 \psi}\left\{\left(d x^{0}\right)^{2}-\left(d x^{1}\right)^{2}-\left(d x^{2}\right)^{2}-\left(d x^{3}\right)^{2}\right\} .
$$

If the vector $\psi_{\mu}$ vanishes identically, then we see from equations (2.14) or (3.6) that the space is flat. Hence the first type of space with teleparallelism is

$$
d s^{2}=\left(d x^{0}\right)^{2}-\left(d x^{1}\right)^{2}-\left(d x^{2}\right)^{2}-\left(d x^{3}\right)^{2} ; \quad \psi_{\mu} \equiv 0 .
$$

§4. Soldtion of the Field Equations-II.

We now assumc that $\psi$ is not a constant and proceed to integrate the equations (2.12). These equations are integrable. The necessary and sufficient condition for this is

$$
\psi_{, \mu \nu \sigma}-\psi_{, \mu \sigma \nu}=\psi_{a} R_{, \mu \nu \sigma}^{a}=0 .
$$

From equation (2.14) we find that this condition is satisfied identically.

Let

$$
a=\psi_{\sigma} \psi^{\sigma} \text {, }
$$

then by covariant differentiation, we find

$$
\frac{\partial a}{\partial x^{\mu}}=2 \psi^{\sigma} \psi_{\sigma \mu}=0, \quad \text { by }(2.12)
$$

hence $a$ is a constant.

Equation (3.2) gives

$$
\left\{\begin{array}{l}
\nu \\
\mu \lambda
\end{array}\right\}=\delta_{\mu}^{\nu} \psi_{\lambda}+\delta_{\lambda}^{\nu} \psi_{\mu}-g_{\mu \lambda} g^{\nu \sigma} \psi_{\sigma} .
$$

Hence $\quad \psi_{, \mu \lambda} \equiv \frac{\partial^{2} \psi}{\partial x^{\mu} \partial x^{\lambda}}-2 \frac{\partial \psi}{\partial x^{\mu}} \frac{\partial \psi}{\partial x^{\lambda}}+g_{\mu \lambda} g^{\nu \sigma} \psi_{\nu} \psi_{\sigma}=0$.

Thus $\frac{\hat{\theta}^{2}}{\partial x^{\mu} \partial x^{\lambda}} e^{-2 \psi}=-2 e^{-2 \psi}\left(\begin{array}{c}\partial^{2} \psi \\ \partial x^{\mu} \partial x^{\lambda}\end{array}-2 \frac{\partial \psi}{\partial x^{\mu}} \frac{\partial \psi}{\partial x^{\lambda}}\right)$

$$
=2 a e^{-2 \psi} g_{\mu \lambda}
$$

using equations (4.1) and (4.2). Then using (3.1) we find

$$
\frac{\delta^{2}}{\partial x^{\mu} \partial x^{\lambda}} e^{-2 \psi}=2 a \gamma_{\mu \lambda}
$$


and, on integration, we obtain

$$
e^{-2 \psi}=b+a_{\sigma} x^{\sigma}+a \gamma_{\mu \nu} x^{\mu} x^{\nu},
$$

where $\gamma_{\mu \nu}$ has Galilean values.

When $a$ is zero, and not all the $a_{\sigma}$ are zero, then by a simple transfurmation of coordinates, leaving $\gamma_{\mu \nu} d x^{\mu} d x^{\nu}$ invariant, the function $e^{-2 \psi}$ can be reduced to the form

$$
e^{-2 \psi}=a_{\sigma} x^{\sigma} \text {. }
$$

Thus we find

$$
\begin{aligned}
& \psi_{\sigma}=-\frac{1}{2} a_{\sigma} e^{2 \psi} \\
& \psi^{\sigma}=-\frac{1}{2} e_{\sigma} a_{\sigma}
\end{aligned}
$$

where $e_{\sigma}=\gamma_{\sigma \sigma}$. But since $a=0$ we have

hence

$$
\left(\psi_{0}\right)^{2}-\left(\psi_{1}\right)^{2}-\left(\psi_{2}\right)^{2}-\left(\psi_{3}\right)^{2}=0,
$$

Then by an orthogonal transformation in $x^{1}, x^{2}, x^{3}$ we can reduce $a_{1} x^{1}+a_{2} x^{2}+a_{3} x^{3}$ to the form $a_{0} x^{3}$, and therefore $e^{-2 \psi}$ is expressible in the form

$$
e^{-2 \psi}=a_{0}\left(x^{0}+x^{3}\right) .
$$

It follows that the metric of the space is

$$
\begin{aligned}
& d s^{2}=\frac{1}{a_{0}}\left(x^{0}+x^{3}\right)^{-1}\left\{\left(d x^{0}\right)^{2}-\left(d x^{1}\right)^{2}-\left(d x^{2}\right)^{2}-\left(d x^{3}\right)^{2}\right\} \\
& \text { and } \quad \psi^{0}=-\frac{1}{2} a_{0}, \psi^{1}=\psi^{2}=0, \psi^{3}=\frac{1}{2} a_{0} .
\end{aligned}
$$

We here observe that it is only when the metric is indefinite, that there exists a real vector such that $\psi_{\sigma} \psi^{\sigma}=0$.

§5. Solution of the Field Equations-III.

Now consider the case when $a$ is not zero. Then

$$
e^{-2 \psi}=b+a_{\sigma} x^{\sigma}+a\left(x^{0}\right)^{2}-a\left(x^{1}\right)^{2}-a\left(x^{2}\right)^{2}-a\left(x^{3}\right)^{2},
$$

and by a transformation of the type

$$
{ }^{\prime} x^{\mu}=x^{\mu}+c^{\mu}
$$

where $c^{\mu}$ is constant, this is reducible to the form

Then

and

Also by (4.1)

whence

$$
e^{-2 \psi}=b+a \gamma_{\mu \nu}, x^{\mu} x^{\nu}
$$


This last equation can be written in the form

$$
g_{\mu \nu} x^{\mu} x^{\nu}=1 / a .
$$

The invariant $a$ has, as we shall see, the value $-1 / R^{2}$. Thus we find the metric of the space in the form

$$
\begin{aligned}
d s^{2} & =-R^{2} \frac{\left(d x^{0}\right)^{2}-\left(d x^{1}\right)^{2}-\left(d x^{2}\right)^{2}-\left(d x^{3}\right)^{2}}{\left(x^{0}\right)^{2}-\left(x^{1}\right)^{2}-\left(x^{2}\right)^{2}-\left(x^{3}\right)^{2}} \\
\psi^{\sigma} & =x^{\sigma} / R^{2} .
\end{aligned}
$$

To obtain a coordinate system whose physical meaning is more evident, we have merely to effect the transformation

$$
\begin{aligned}
& x^{0}=i R e^{i t / R} \cos \chi \\
& x^{1}=R e^{i t / R} \sin \chi \sin \theta \cos \phi \\
& x^{2}=R e^{i t / R} \sin \chi \sin \theta \sin \phi \\
& x^{3}=R e^{i t / R} \sin \chi \cos \theta ;
\end{aligned}
$$

the metric then takes the form

$$
d s^{2}=d t^{2}-R^{2}\left\{d \chi^{2}+\sin ^{2} \chi\left(d \theta^{2}+\sin ^{2} \theta d \phi^{2}\right)\right\} .
$$

This is Einstein's cylindrical world in its usual form. . The equation (III) however, exhibits it in a form which is symmetrical in all its coordinates, where we have, too, the advantage that the null geodesics of the manifold are

$$
x^{1}=a x^{0}+a^{\prime}, x^{2}=b x^{0}+b^{\prime}, x^{3}=c x^{0}+c^{\prime},
$$

where $a^{2}+b^{2}+c^{2}=1$. In this coordinate system we find from (5.4) that $\psi=-i t / R$. 